\title{
ON THE SETS OF REAL NUMBERS RECOGNIZED BY FINITE AUTOMATA IN MULTIPLE BASES*
}

\author{
BERNARD BOIGELOT $^{a}$, JULIEN BRUSTEN $^{b}$, AND VÉRONIQUE BRUYĖRE $^{c}$ \\ ${ }^{a, b}$ Université de Liège, Institut Montefiore, B28, B-4000 Liège, Belgium \\ e-mail address: \{boigelot,brusten\}@montefiore.ulg.ac.be \\ ${ }^{c}$ Université de Mons-Hainaut, Avenue du Champ de Mars, 6, B-7000 Mons, Belgium \\ e-mail address: veronique.bruyere@umh.ac.be
}

\begin{abstract}
This article studies the expressive power of finite automata recognizing sets of real numbers encoded in positional notation. We consider Muller automata as well as the restricted class of weak deterministic automata, used as symbolic set representations in actual applications. In previous work, it has been established that the sets of numbers that are recognizable by weak deterministic automata in two bases that do not share the same set of prime factors are exactly those that are definable in the first order additive theory of real and integer numbers. This result extends Cobham's theorem, which characterizes the sets of integer numbers that are recognizable by finite automata in multiple bases.

In this article, we first generalize this result to multiplicatively independent bases, which brings it closer to the original statement of Cobham's theorem. Then, we study the sets of reals recognizable by Muller automata in two bases. We show with a counterexample that, in this setting, Cobham's theorem does not generalize to multiplicatively independent bases. Finally, we prove that the sets of reals that are recognizable by Muller automata in two bases that do not share the same set of prime factors are exactly those definable in the first order additive theory of real and integer numbers. These sets are thus also recognizable by weak deterministic automata. This result leads to a precise characterization of the sets of real numbers that are recognizable in multiple bases, and provides a theoretical justification to the use of weak automata as symbolic representations of sets.
\end{abstract}

\section{INTRODUCTION}

By using positional notation, real numbers can be encoded as infinite words over an alphabet composed of a fixed number of digits, with an additional symbol for separating their

1998 ACM Subject Classification: F.1.1; F.4.1; F.4.3.

Key words and phrases: automata, real numbers, mixed real-integer arithmetic, Cobham's theorem.

* A preliminary version of this work appears in the proceedings of the 35th International Colloquium on Automata, Languages and Programming (ICALP'08). This work is supported by the Interuniversity Attraction Poles program MoVES of the Belgian Federal Science Policy Office, and by the grant 2.4530.02 of the Belgian Fund for Scientific Research (F.R.S.-FNRS).

${ }^{b}$ Research fellow ("Aspirant") of the Belgian Fund for Scientific Research (F.R.S.-FNRS).

님
DOI:10.2168/LMCS-6 (1:6) 2010

(C) B. Boigelot, J. Brusten, and V. Bruyère (c) Creative Commons 
integer and fractional parts. This encoding scheme maps sets of numbers onto languages that describe those sets unambiguously.

This article studies the sets of real numbers whose encodings can be accepted by finite automata. The motivation is twofold. First, since regular languages enjoy good closure properties under a large range of operators, automata provide powerful theoretical tools for establishing the decidability of arithmetic theories. In particular, it is known that the sets of numbers that are definable in the first-order additive theory of integers $\langle\mathbb{Z},+,<\rangle$, also called Presburger arithmetic, are encoded by regular finite-word languages [Büc62, BHMV94. This result translates into a simple procedure for deciding the satisfiability of Presburger formulas. Moving to infinite-word encodings and $\omega$-regular languages, it can be extended to sets of real numbers definable in $\langle\mathbb{R}, \mathbb{Z},+,<\rangle$, i.e., the first-order additive theory of real and integer variables [BBR97, BRW98, BJW05].

The second motivation is practical. Since finite automata are objects that are easily manipulated algorithmically, they can be used as actual data structures for representing symbolically sets of values. This idea has successfully been exploited in the context of computer-aided verification, leading to representations suited for the sets of real and integer vectors handled during symbolic state-space exploration [WB95, Boi98, BJW05, EK06. A practical limitation of this approach is the high computational cost of some operations involving infinite-word automata, in particular language complementation [Saf88, Var07]. However, it has been shown that a restricted form of automata, weak deterministic ones, actually suffices for handling the sets definable in $\langle\mathbb{R}, \mathbb{Z},+,<\rangle$ BJW05. Weak automata can be manipulated with essentially the same cost as finite-word ones Wil93, which alleviates the problem and leads to an effective representation system.

Whether a set of numbers can be recognized by an automaton generally depends on the chosen encoding base. For integer numbers, it is known that a set $S \subseteq \mathbb{Z}$ is recognizable in a base $r>1$ iff it is definable in the theory $\left\langle\mathbb{Z},+,<, V_{r}\right\rangle$, where $V_{r}$ is a base-dependent function [BHMV94] that returns the highest integer power of $r$ dividing its argument. Furthermore, the well-known Cobham's theorem states that if a set $S \subseteq \mathbb{N}$ is simultaneously recognizable in two bases $r>1$ and $s>1$ that are multiplicatively independent, i.e., such that $r^{p} \neq s^{q}$ for all $p, q \in \mathbb{N}_{>0}$, then $S$ is ultimately periodic, i.e., it differs from a periodic subset of $\mathbb{N}$ only by a finite set Cob69. As a corollary of Cobham's theorem, a subset of $\mathbb{Z}$ that is recognizable in two multiplicatively independent bases is definable in $\langle\mathbb{Z},+,<\rangle$ BHMV94, from which it follows that it is recognizable in every base. Our aim is to generalize as completely as possible this result to automata recognizing real numbers, by precisely characterizing the sets that are recognizable in multiple bases. We first consider the case, relevant for practical applications, of weak deterministic automata. In previous work, it has been established that a set of real numbers is simultaneously recognizable by weak deterministic automata in two bases that do not share the same set of prime factors iff this set is definable in $\langle\mathbb{R}, \mathbb{Z},+,<\rangle$ BB09]. As a first contribution, we extend this result to pairs of multiplicatively independent bases. Since recognizability in two multiplicatively dependent bases is equivalent to recognizability in only one of them [BRW98, this result provides a complete characterization of the sets that are recognizable in multiple bases by weak deterministic automata.

Then, we move to sets recognized by Muller automata. We establish that there exists a set of real numbers recognizable in two multiplicatively independent bases that share the same set of prime factors, but that is not definable in $\langle\mathbb{R}, \mathbb{Z},+,<\rangle$. This shows that Cobham's theorem does not directly generalize to Muller automata recognizing sets of real 
numbers. Finally, we establish that a set $S \subseteq \mathbb{R}$ is simultaneously recognizable in two bases that do not share the same set of prime factors iff $S$ is definable in $\langle\mathbb{R}, \mathbb{Z},+,<\rangle$. As a corollary, such a set must then be recognizable by a weak deterministic automaton. Our result thus provides a theoretical justification to the use of weak automata, by showing that their expressive power corresponds precisely to the sets of reals recognizable by infinite-word automata in every encoding base.

\section{BASIC NOTIONS}

2.1. Encodings of numbers. Let $r \in \mathbb{N}_{>1}$ be an integer numeration base and let $\Sigma_{r}=$ $\{0, \ldots, r-1\}$ be the corresponding set of digits. We encode a real number $x$ in base $r$, most significant digit first, by words of the form $w_{I} \star w_{F}$, where $w_{I} \in \Sigma_{r}^{*}$ encodes an integer part $x_{I} \in \mathbb{Z}$ of $x$ and $w_{F} \in \Sigma_{r}^{\omega}$ encodes a fractional part $x_{F} \in[0,1]$. Note that the decomposition of $x$ into $x_{I}$ and $x_{F}$ is not necessarily unique, e.g., $x=3$ gives either $x_{I}=3$ and $x_{F}=0$, or $x_{I}=2$ and $x_{F}=1$. Negative integer numbers are represented by their $r$ 's-complement, i.e., the encodings of $x_{I} \in \mathbb{Z}_{<0}$ are formed by the last $p$ digits of the encodings of $r^{p}+x_{I}$. The length $p$ of $w_{I}$ is not fixed but has to be large enough for $-r^{p-1} \leq x_{I}<r^{p-1}$ to hold; thus, the most significant digit of an encoding is equal to 0 for positive integer parts and to $r-1$ for negative ones [BBR97]. As a consequence, the set of valid encodings of numbers in base $r$ forms the language $\{0, r-1\} \Sigma_{r}^{*} \star \Sigma_{r}^{\omega}$. Some numbers have two distinct encodings with the same integer-part length, e.g., in base 10, the number $11 / 2$ admits the encodings $0^{+} 5 \star 50^{\omega}$ and $0^{+} 5 \star 49^{\omega}$. Such encodings are called dual. For a word $w=b_{p-1}^{I} b_{p-2}^{I} \ldots b_{1}^{I} b_{0}^{I} \star b_{1}^{F} b_{2}^{F} b_{3}^{F} \ldots \in\{0, r-1\} \Sigma_{r}^{*} \star \Sigma_{r}^{\omega}$, we denote by $[w]_{r}$ the real number encoded by $w$ in base $r$, i.e.,

$$
[w]_{r}=\sum_{i=0}^{p-2} b_{i}^{I} r^{i}+\sum_{i>0} b_{i}^{F} r^{-i}+\left\{\begin{array}{cl}
0 & \text { if } b_{p-1}^{I}=0, \\
-r^{p-1} & \text { if } b_{p-1}^{I}=r-1 .
\end{array}\right.
$$

For finite words $w \in \Sigma_{r}^{*}$, we denote by $[w]_{r}$ the natural number encoded by $w$, i.e., $[w]_{r}=$ $\left[0 w \star 0^{\omega}\right]_{r}$.

It is known HW85] that a word $w \in\{0, r-1\} \Sigma_{r}^{*} \star \Sigma_{r}^{\omega}$ is ultimately periodic, i.e., of the form $\{0, r-1\} u_{1} \star u_{2} u_{3}^{\omega}$ with $u_{1}, u_{2} \in \Sigma_{r}^{*}$ and $u_{3} \in \Sigma_{r}^{+}$, if and only if $[w]_{r}$ is rational. The word $u_{3}$ is then called a period of $w$.

2.2. Real Number Automata. For a set $S \subseteq \mathbb{R}$, we denote by $L_{r}(S)$ the language of all the base- $r$ encodings of the elements of $S$. If $L_{r}(S)$ is $\omega$-regular, then it can be accepted by a (non-unique) infinite-word automaton, called a Real Number Automaton (RNA), recognizing $S$. Such a set $S$ is then said to be $r$-recognizable. RNA can be generalized into Real Vector Automata (RVA), suited for subsets of $\mathbb{R}^{n}$, with $n>0$ [BBR97].

RNA have originally been defined as Büchi automata [BBR97. In this article, we will instead consider them to be deterministic Muller automata. This adaptation can be made without loss of generality, since both classes of automata share the same expressive power [McN66, PP04]. The fact that RNA have a deterministic transition relation will simplify technical developments.

The $r$-recognizable sets of real numbers are precisely described by the following result. This logical characterization will often be used in this article. 
Theorem 2.1 ([BRW98]). Let $r \in \mathbb{N}_{>1}$ be a base. A subset of $\mathbb{R}$ is $r$-recognizable iff it is definable in the first-order theory $\left\langle\mathbb{R}, \mathbb{Z},+,<, X_{r}\right\rangle$, where $X_{r}(x, u, k)$ is a base-dependent predicate that holds whenever $u$ is an integer power of $r$, and there exists an encoding of $x$ in which the digit at the position specified by $u$ is equal to $k$.

It is known that the full expressive power of infinite-word automata is not needed for representing the subsets of $\mathbb{R}$ that are definable in $\langle\mathbb{R}, \mathbb{Z},+,<\rangle$. The following theorem establishes that such sets can be recognized by weak deterministic automata, i.e., deterministic Büchi automata such that each strongly connected component of their transition graph contains either only accepting or only non-accepting states. A set recognized by a weak deterministic automaton in base $r$ is said to be weakly $r$-recognizable, and such an automaton is then called a weak RNA.

Theorem 2.2 ([BJW05]). If a subset of $\mathbb{R}$ is definable in the first-order theory $\langle\mathbb{R}, \mathbb{Z},+,<\rangle$, then it is weakly $r$-recognizable in every base $r \in \mathbb{N}_{>1}$.

2.3. Topology. In this section, we recall some notions about topology, which is a useful tool for reasoning about the properties of sets of words and numbers [PP04].

2.3.1. General concepts. Given a set $S$, either of words or of numbers, a distance $d(x, y)$ defined on this set induces a metric topology on subsets of $S$. A neighborhood $N_{\varepsilon}(x)$ of a point $x \in S$ with respect to $\varepsilon \in \mathbb{R}_{>0}$ is the set $N_{\varepsilon}(x)=\{y \mid d(x, y)<\varepsilon\}$. A set $C \subseteq S$ is said to be open if for all $x \in C$, there exists $\varepsilon>0$ such that $N_{\varepsilon}(x) \subseteq C$. A closed set is a set whose complement with respect to $S$ is open, or, equivalently, a set that contains the limits of all its converging sequences of elements. The following notations will be used:

- $F$ is the class of closed sets,

- $G$ is the class of open sets,

- $F_{\sigma}$ is the class of countable unions of closed sets,

- $G_{\delta}$ is the class of countable intersections of open sets.

Other classes can be defined from these notations: The class $\mathcal{B}(F)=\mathcal{B}(G)$ contains the finite Boolean combinations of open and closed sets, whereas $F_{\sigma} \cap G_{\delta}$ is the class of sets that can be expressed as countable unions of closed sets as well as countable intersections of open sets.

Those classes of sets are the first levels of the Borel hierarchy. In a metric topology, this hierarchy states that $F$ and $G$ are subclasses of $\mathcal{B}(F)=\mathcal{B}(G)$, which is itself a subclass of $F_{\sigma} \cap G_{\delta}$.

2.3.2. Topology of $\omega$-words. Given a base $r \in \mathbb{N}_{>1}$ and the alphabet $\Sigma_{r} \cup\{\star\}$, we define the following distance relation between infinite words over this alphabet:

$$
d\left(w, w^{\prime}\right)=\left\{\begin{array}{cl}
\frac{1}{\left|\operatorname{common}\left(w, w^{\prime}\right)\right|+1} & \text { if } w \neq w^{\prime} \\
0 & \text { if } w=w^{\prime}
\end{array}\right.
$$

where $\left|\operatorname{common}\left(w, w^{\prime}\right)\right|$ denotes the length of the longest common prefix of $w$ and $w^{\prime}$. This distance induces a topology on $\left(\Sigma_{r} \cup\{\star\}\right)^{\omega}$. 
We say that a $\omega$-language $L \subseteq\left(\Sigma_{r} \cup\{\star\}\right)^{\omega}$ satisfies the dense oscillating sequence property if, $w_{1}, w_{2}, w_{3}, \ldots$ being $\omega$-words and $\varepsilon_{1}, \varepsilon_{2}, \varepsilon_{3}, \ldots$ being distances, one has that

$$
\exists w_{1} \forall \varepsilon_{1} \exists w_{2} \forall \varepsilon_{2} \exists w_{3} \forall \varepsilon_{3} \cdots
$$

such that $d\left(w_{i}, w_{i+1}\right) \leq \varepsilon_{i}$ for all $i \geq 1, w_{i} \in L$ for all odd $i$, and $w_{i} \notin L$ for all even $i$ [BJW05].

It has been established [MS97] that weak deterministic automata accept exactly the $\omega$-regular languages that belong to the topological class $F_{\sigma} \cap G_{\delta}$.

It is also known [BJW05] that the $\omega$-regular languages that satisfy the dense oscillating sequence property cannot be accepted by weak deterministic automata.

2.3.3. Topology of real numbers. We consider the topology on the sets of real numbers induced by the distance relation defined by $d(x, y)=|x-y|$.

In this topology, a notion of dense oscillating sequence can be defined in the same way as for $\omega$-words: We say that a set $S \subseteq \mathbb{R}$ satisfies the dense oscillating sequence property if, $x_{1}, x_{2}, x_{3}, \ldots$ being real numbers and $\varepsilon_{1}, \varepsilon_{2}, \varepsilon_{3}, \ldots$ being distances, one has that

$$
\exists x_{1} \forall \varepsilon_{1} \exists x_{2} \forall \varepsilon_{2} \exists x_{3} \forall \varepsilon_{3} \cdots
$$

such that $d\left(x_{i}, x_{i+1}\right) \leq \varepsilon_{i}$ for all $i \geq 1, x_{i} \in S$ for all odd $i$, and $x_{i} \notin S$ for all even $i$.

We have the following theorem.

Theorem 2.3. Let $r \in \mathbb{N}_{>1}$ be a base. The r-recognizable sets $S \subseteq \mathbb{R}$ that satisfy the dense oscillating sequence property are not weakly r-recognizable.

Proof. Consider a $r$-recognizable set $S \subseteq \mathbb{R}$ satisfying the dense oscillating sequence property. It is sufficient to establish that $L_{r}(S)$ satisfies the dense oscillating sequence property as well.

Recall that each real number admits multiple encodings. First, the first digit of an encoding can be repeated at will. Second, for a given length of the integer part (assumed to be sufficiently large), a number admits either one encoding, or two (dual) ones.

Let $S_{1}, S_{2} \subseteq \mathbb{R}$ be sets of numbers such that $S_{1} \cap S_{2}=\emptyset$. Consider any number $x_{1} \in S_{1}$ for which there exist arbitrarily close numbers in $S_{2}$. Then, there exists an encoding $w_{1}$ of $x_{1}$ for which there exist arbitrarily close encodings $w_{2}$ of numbers $x_{2}$ of $S$. We can ask more: There exists an encoding $w_{1}$ of $x_{1}$ for which there exist arbitrarily close encodings $w_{2}$ of numbers $x_{2}$ of $S_{2}$, including the dual encodings with the same integer part length as $w_{1}$, if any. Formally, $\left(x_{1} \in S_{1} \wedge(\forall \varepsilon>0)\left(\exists x_{2} \in S_{2}\right)\left(d\left(x_{1}, x_{2}\right)<\varepsilon\right)\right) \Rightarrow\left(\exists w_{1}\right)\left(\left[w_{1}\right]_{r}=\right.$ $x_{1} \wedge\left(\forall \varepsilon^{\prime}>0\right)\left(\exists x_{2} \in S_{2}\right)\left(\left(\exists w_{2}\right)\left(\left[w_{2}\right]_{r}=x_{2} \wedge\left|w_{1}\right|_{I}=\left|w_{2}\right|_{I}\right) \wedge\left(\forall w_{2}\right)\left(\left[w_{2}\right]_{r}=x_{2} \wedge\left|w_{1}\right|_{I}=\right.\right.$ $\left.\left.\left.\left|w_{2}\right|_{I} \Rightarrow d\left(w_{1}, w_{2}\right)<\varepsilon^{\prime}\right)\right)\right)$, where $|w|_{I}$ denotes the integer part length of the encoding $w$.

By hypothesis, there exists $x_{1} \in S$ such that $\forall \varepsilon_{1} \exists x_{2} \forall \varepsilon_{2} \exists x_{3} \forall \varepsilon_{3} \cdots, d\left(x_{i}, x_{i+1}\right) \leq \varepsilon_{i}$ for all $i \geq 1, x_{i} \in S$ for all odd $i$, and $x_{i} \notin S$ for all even $i$. We choose $S_{1}=S$, and define $S_{2}$ as the subset of $\bar{S}$ whose elements $x_{2}$ satisfy $\forall \varepsilon_{2} \exists x_{3} \forall \varepsilon_{3} \exists x_{4} \forall \varepsilon_{4} \cdots, d\left(x_{i}, x_{i+1}\right) \leq \varepsilon_{i}$ for all $i \geq 2, x_{i} \in S$ for all odd $i$, and $x_{i} \notin S$ for all even $i$. By the previous property, there exists an encoding $w_{1}$ of $x_{1}$ such that for arbitrarily small $\varepsilon^{\prime}>0$, there exists an element $x_{2}$ of $S_{2}$ whose all encodings $w_{2}$ satisfy $d\left(w_{1}, w_{2}\right)<\varepsilon^{\prime}$, provided that they share the same integerpart length as $w_{1}$. Moreover, there exists at least one such encoding $w_{2}$. By applying a similar reasoning to $x_{2}, x_{3}, x_{4}, \ldots$, one obtains $\exists w_{1} \forall \varepsilon_{1}^{\prime} \exists w_{2} \forall \varepsilon_{2}^{\prime} \exists w_{3} \forall \varepsilon_{3}^{\prime} \cdots, d\left(w_{i}, w_{i+1}\right) \leq \varepsilon_{i}^{\prime}$ for all $i \geq 1, w_{i} \in L_{r}(S)$ for all odd $i$, and $w_{i} \notin L_{r}(S)$ for all even $i$. It follows that the language $L_{r}(S)$ satisfies the dense oscillating sequence property. 
2.3.4. Links between the topology of $\omega$-words and the topology of real numbers. In this section, the notations $F_{\sigma}, G_{\delta}$ and $F_{\sigma} \cap G_{\delta}$ (resp. $\mathrm{F}_{\sigma}, \mathrm{G}_{\delta}$ and $\mathrm{F}_{\sigma} \cap \mathrm{G}_{\delta}$ ) will be used when dealing with the topology of $\omega$-words (resp. real numbers).

Lemma 2.4. Let $r \in \mathbb{N}_{>1}$ be a base, and let $L \subseteq\left(\Sigma_{r} \cup\{\star\}\right)^{\omega}$ be a language. If $L$ belongs to $F_{\sigma}$, then the set of real numbers that have an encoding in $L$ belongs to $F_{\sigma}$.

Proof. Let $W_{j}$ be the language $\{0, r-1\}\{0, \ldots, r-1\}^{j} \star\left(\Sigma_{r} \cup\{\star\}\right)^{\omega}$ with $j \in \mathbb{N}$. This language is open for all $j$. Since $L$ belongs to $F_{\sigma}$, it can be expressed as $L=\bigcup_{i \in \mathbb{N}} F_{i}$, where each $F_{i}$ is closed. The language $\bigcup_{i \in \mathbb{N}} \bigcup_{j \in \mathbb{N}}\left(F_{i} \cap W_{j}\right)$ is a sublanguage of $L$ such that the language of valid encodings it contains is exactly the language of valid encodings that belong to $L$. When $i$ and $j$ are fixed, the set $F_{i} \cap W_{j}$ is the intersection of a closed and an open set; hence, it belongs to $F_{\sigma}$ and is thus a countable union of closed sets: $F_{i} \cap W_{j}=\bigcup_{k \in \mathbb{N}} L_{i, j, k}$.

For each of these closed sets $L_{i, j, k}$, define $S_{i, j, k} \subseteq \mathbb{R}$ as the set of numbers that have at least one encoding in $L_{i, j, k}$. The set $S_{i, j, k}$ is closed. Indeed, suppose that $S_{i, j, k}$ is not closed. Thus, there exists a converging sequence of points of $S_{i, j, k}$ whose limit $x$ does not belong to $S_{i, j, k}$. If this sequence contains infinitely many points greater than $x$, one extracts its subsequence composed of those points. Otherwise, one extracts the subsequence composed of its points that are lower than $x$. Each of the points of $S_{i, j, k}$ has at least one encoding in $F_{i} \cap W_{j}$. Since the valid encodings in $F_{i} \cap W_{j}$ have the same integer part length, the converging subsequence of points of $S_{i, j, k}$ is mapped to a converging sequence of words encoding those points. Since $L_{i, j, k}$ is closed, it contains the limit of its converging sequences, hence the limit $x$ of the converging sequence of points of $S_{i, j, k}$ has an encoding in $L_{i, j, k}$, which leads to a contradiction since this limit would be in $S_{i, j, k}$.

It follows that the set of real numbers that have an encoding in $L$ is a countable union $\bigcup_{(i, j, k) \in \mathbb{N}^{3}} S_{i, j, k}$ of closed sets in $\mathbb{R}$, and thus belongs to $\mathrm{F}_{\sigma}$.

Lemma 2.5. Let $S \subseteq \mathbb{R}$, and $r \in \mathbb{N}_{>1}$ be a base. The set $S$ belongs to $\mathrm{F}_{\sigma} \cap \mathrm{G}_{\delta}$ iff the language $L_{r}(S)$ belongs to $F_{\sigma} \cap G_{\delta}$.

Proof. It is known [BJW05] that if a set $S \subseteq \mathbb{R}$ belongs to $\mathrm{F}_{\sigma} \cap \mathrm{G}_{\delta}$, then the language $L_{r}(S)$ belongs to $F_{\sigma} \cap G_{\delta}$.

If $L_{r}(S)$ belongs to $F_{\sigma} \cap G_{\delta}$, then it belongs in particular to $F_{\sigma}$. By Lemma 2.4, $S$ then belongs to $\mathrm{F}_{\sigma}$. On the other hand, $L_{r}(S)$ belongs to $G_{\delta}$. It follows that the complement of $L_{r}(S)$ belongs to $F_{\sigma}$. By Lemma 2.4, the set of real numbers that have an encoding in this language belongs to $\mathrm{F}_{\sigma}$, which implies that $S$ belongs to $\mathrm{G}_{\delta}$.

In the sequel, we will need to apply transformations to sets represented by RNA (or weak RNA), or to the chosen encoding base.

Theorem 2.6. Let $S \subseteq \mathbb{R}, r \in \mathbb{N}_{>1}$, and $a, b \in \mathbb{Q}$. If $S$ is (resp. weakly) r-recognizable then the sets $a S+b$ and $S \cap[a, b]$ are (resp. weakly) r-recognizable as well.

Proof. If $S$ is $r$-recognizable, then it is definable in $\left\langle\mathbb{R}, \mathbb{Z},+,<, X_{r}\right\rangle$ by Theorem 2.1, and so are the sets $a S+b$ and $S \cap[a, b]$, that thus are both $r$-recognizable.

If $S$ is weakly $r$-recognizable, then the language $L_{r}(S)$ belongs to the class $F_{\sigma} \cap G_{\delta}$. By Lemma 2.5, the set $S$ belongs to the class $\mathrm{F}_{\sigma} \cap \mathrm{G}_{\delta}$, and so are the sets $a S+b$ and $S \cap[a, b]$. Since these sets are $r$-recognizable by the first part of the proof, it follows from [MS97 that they are also weakly $r$-recognizable. 
Theorem 2.7. Let $S \subseteq \mathbb{R}, r \in \mathbb{N}_{>1}$, and $l \in \mathbb{N}_{>0}$. The set $S$ is (resp. weakly) r-recognizable iff it is (resp. weakly) $r^{l}$-recognizable.

Proof. If $S$ is $r$-recognizable, then the result is a consequence of Theorem 2.1, since the predicate $X_{r}(x, u, k)$ can be expressed in terms of $X_{r l}(x, u, k)$, and reciprocally. Indeed, testing the value of the digit at a given position in an encoding in base $r^{l}$ can be reduced to the test of $l$ digits in base $r$, and conversely.

If $S$ is weakly $r$-recognizable, then $L_{r}(S)$ belongs to the class $F_{\sigma} \cap G_{\delta}$. By Lemma 2.5, $S$ belongs to the class $\mathrm{F}_{\sigma} \cap \mathrm{G}_{\delta}$, and $L_{r l}(S)$ belongs to the class $F_{\sigma} \cap G_{\delta}$. Since $S$ is $r^{l}-$ recognizable, $S$ is weakly $r^{l}$-recognizable. The case of a $r^{l}$-recognizable set $S$ is handled in the same way.

\section{PRIOR RESUlts AND OBJECTIVES}

This article is aimed at characterizing precisely the conditions under which a set of real numbers is recognizable, or weakly recognizable, in multiple bases. We start by summarizing some known results.

First, the case of sets of integer numbers is handled by the following result, which is a direct corollary of the well-known Cobham's theorem. Note that for sets of integer numbers, the notions of $r$-recognizability and weak $r$-recognizability coincide, and correspond to the existence of a finite-word automaton accepting only the integer part of encodings.

Theorem 3.1 ([Cob69, BHMV94]). Let $r, s \in \mathbb{N}_{>1}$ be bases that are multiplicatively independent, i.e., such that $r^{p} \neq s^{q}$ for all $p, q \in \mathbb{N}_{>0}$. A set $S \subseteq \mathbb{Z}$ is both $r$-and s-recognizable iff it is definable in the first-order theory $\langle\mathbb{Z},+,<\rangle$.

If $r, s \in \mathbb{N}_{>1}$ are multiplicatively dependent, then a set $S \subseteq \mathbb{Z}$ is $r$-recognizable iff it is $s$-recognizable, as a consequence of Theorem 2.7. It follows that Theorem 3.1 fully characterizes recognizability in multiple bases for sets of integer numbers.

Next, for sets of real numbers recognized by weak automata, we have the following result.

Theorem 3.2 ([BB09]). Let $r, s \in \mathbb{N}_{>1}$ be bases that do not share the same set of prime factors. A set $S \subseteq \mathbb{R}$ is both weakly $r$ - and weakly s-recognizable iff it is definable in the first-order theory $\langle\mathbb{R}, \mathbb{Z},+,<\rangle$.

In this paper, we extend Theorem 3.2 in two ways. First, we will show in Section 5 that this result also holds for multiplicatively independent bases, which weakens the hypotheses of the theorem and brings its statement closer to Theorem 3.1. Formally, we will prove the following theorem.

Theorem 3.3. Let $r, s \in \mathbb{N}_{>1}$ be two multiplicatively independent bases. A set $S \subseteq \mathbb{R}$ is both weakly $r$-and weakly s-recognizable iff it is definable in the first-order theory $\langle\mathbb{R}, \mathbb{Z},+,<\rangle$.

Second, we will establish in Section 6 that a similar result holds for recognizable (as opposed to weakly recognizable) sets of real numbers. Formally, we will prove the following theorem.

Theorem 3.4. Let $r, s \in \mathbb{N}_{>1}$ be two bases that do not share the same set of prime factors. A set $S \subseteq \mathbb{R}$ is both $r$ - and s-recognizable iff it is definable in the first-order theory $\langle\mathbb{R}, \mathbb{Z},+,<\rangle$. 
In this particular case, we will also show that considering bases with different sets of prime factors is essential, and that multiplicatively independent bases do not lead to a similar property.

Before proving Theorems 3.3 and 3.4, we show in the next section that these problems can be reduced to simpler ones.

\section{Problem Reductions}

In the next sections, we will consider sets $S \subseteq \mathbb{R}$ that are simultaneously recognizable, either by RNA or by weak RNA, in two bases $r$ and $s$ that either are multiplicatively independent, or have different sets of prime factors. We will then tackle the problem of proving that such sets are definable in $\langle\mathbb{R}, \mathbb{Z},+,<\rangle$. In this section, we reduce this problem, by restricting the domain to the interval $[0,1]$, and introducing the notion of boundary point.

4.1. Reduction to $[0,1]$. This section is adapted from [BB09]. Let $S \subseteq \mathbb{R}$ be a set of real numbers. The set $S$ can be decomposed into a countable union

$$
\bigcup_{i \in \mathbb{Z}}\left(\{i\}+S_{i}^{F}\right)
$$

where for all $i, S_{i}^{F} \subseteq[0,1]$ is the set of fractional parts that can be added to the integer $i$ to obtain an element $x \in S$.

If we decompose the set $\mathbb{Z}$ into equivalence classes $S_{1}^{I}, S_{2}^{I}, S_{3}^{I}, \ldots$ such that two integers $i$ and $j$ are in the same equivalence class iff the sets $S_{i}^{F}$ and $S_{j}^{F}$ are identical, then this union becomes a (finite or infinite) union

$$
\bigcup_{i}\left(S_{i}^{I}+S_{i}^{F}\right)
$$

Assume now that $S$ is recognizable by a (resp. weak) RNA $\mathcal{A}$ in some base $r \in \mathbb{N}_{>1}$. Recall that $\mathcal{A}$ has a deterministic transition relation. For each encoding of each possible value $x_{I} \in \mathbb{Z}$, the path in $\mathcal{A}$ that reads this encoding followed by the separator $\star$ leads to a state $q$ accepting a language $L_{q}$. The language $0^{+} \star L_{q}$ encodes the set of all fractional parts $x_{F}$ that can be associated to $x_{I}$, i.e., the set $S_{q}=\left\{x_{F} \in[0,1] \mid x_{I}+x_{F} \in S\right\}$. Note that the dual encodings of 0 and 1 may be missing, but this is not problematic.

Such states $q$ are in a finite number $n$, and can w.l.o.g. be supposed to accept languages that are pairwise different (otherwise, it suffices to modify the destinations of the transitions labeled by $\star$ that lead to redundant states). Assuming w.l.o.g. that the languages accepted from every state are not empty, it follows that the languages $L_{q}$ are in the same finite number $n$, and so are the sets $S_{q}$. The sets $S_{q}$ correspond exactly to those of the sets $S_{i}^{F}$ that are not empty. Hence, the number $n$ of sets $S_{q}$ is independent from the representation base.

The set $S$ can thus be decomposed into a finite union

$$
\bigcup_{i=1}^{n}\left(S_{i}^{I}+S_{i}^{F}\right),
$$

where the sets $S_{i}^{I} \subseteq \mathbb{Z}$ are non-empty and pairwise distinct, and the sets $S_{i}^{F} \subseteq[0,1]$ are non-empty and pairwise different. Furthermore, each set $S_{i}^{I}$ is recognizable by a finite-word 
automaton in every base in which $S$ is recognizable, and each set $S_{i}^{F}$ is (resp. weakly) recognizable in every base in which $S$ is (resp. weakly) recognizable1.

Assume now that $S \subseteq \mathbb{R}$ is simultaneously (resp. weakly) $r$ - and $s$ - recognizable, with respect to bases $r$ and $s$ that are multiplicatively independent. By Theorem 3.1, each set $S_{i}^{I}$ is thus definable in $\langle\mathbb{Z},+,<\rangle$. This reduces the problem of establishing that $S$ is definable in $\langle\mathbb{R}, \mathbb{Z},+,<\rangle$ to the same problem for each set $S_{i}^{F}$. Since we have $S_{i}^{F} \subseteq[0,1]$ for all $i$, the problem has thus been reduced from the domain $\mathbb{R}$ to the interval $[0,1]$.

4.2. Boundary points. A point $x \in \mathbb{R}$ is a boundary point of a set $S \subseteq \mathbb{R}$ iff all its neighborhoods contain at least one point from $S$ as well as one from its complement $\bar{S}=$ $\mathbb{R} \backslash S$.

Lemma 4.1. Let $r \in \mathbb{N}_{>1}$ be a base. If a set $S \subseteq \mathbb{R}$ is $r$-recognizable, then the set $B_{S}$ of boundary points of $S$ is $r$-recognizable.

Proof. Since $S$ is $r$-recognizable, it is definable in $\left\langle\mathbb{R}, \mathbb{Z},+,<, X_{r}\right\rangle$ by Theorem 2.1. It is sufficient to show that $B_{S}$ is definable in $\left\langle\mathbb{R}, \mathbb{Z},+,<, X_{r}\right\rangle$. A formula defining $B_{S}$ in this theory is

$$
\left\{x \in \mathbb{R} \mid\left(\forall \varepsilon \in \mathbb{R}_{>0}\right)(\exists y, z \in \mathbb{R})(y \in S \wedge z \notin S \wedge|x-y|<\varepsilon \wedge|x-z|<\varepsilon\} .\right.
$$

Lemma 4.2. Let $r \in \mathbb{N}_{>1}$ be a base. If a r-recognizable set $S \subseteq \mathbb{R}$ has only finitely many boundary points, then it is definable in the first-order theory $\langle\mathbb{R}, \mathbb{Z},+,<\rangle$.

Proof. If $S \subseteq \mathbb{R}$ has only finitely many boundary points, then it can be decomposed into a finite union of intervals such that the extremities of these intervals are the boundary points of $S$.

In order to prove that $S$ is definable in $\langle\mathbb{R}, \mathbb{Z},+,<\rangle$, it is sufficient to show that the boundary points of $S$ are rational numbers. Since $S$ is $r$-recognizable, the finite set $B_{S}$ of its boundary points is $r$-recognizable by Lemma 4.1. It follows that its elements are encoded by words accepted by a finite automaton, and that share a finite number of fractional parts. These are necessarily ultimately periodic, from which the elements of $B_{S}$ are rational.

\section{Multiplicatively indePendent Bases}

Let $r, s \in \mathbb{N}_{>1}$ be two multiplicatively independent bases. The first aim of this section is to prove Theorem 3.3 i.e., to establish that the subsets of $\mathbb{R}$ that are both weakly $r$ - and weakly $s$-recognizable are exactly those that are definable in $\langle\mathbb{R}, \mathbb{Z},+,\langle\rangle$. Then, a second goal will be to show that the subsets of $\mathbb{R}$ that are both $r$ - and $s$-recognizable do not enjoy the same property.

Thanks to the reduction discussed in Section 4.1, it is sufficient to prove these results for sets restricted to the interval $[0,1]$. Besides, Lemma 4.2 implies that, in order to show that a recognizable set is definable in $\langle\mathbb{R}, \mathbb{Z},+,\langle\rangle$, it suffices to prove that it admits only a finite number of boundary points.

\footnotetext{
${ }^{1}$ Indeed, in any (resp. weak) RNA recognizing the set $S$, there exists a state $q$ accepting a language $L_{q}$ such that the language encoding $S_{i}^{F}$ is $0^{+} \star L_{q}$. In order for such a language to contain all encodings of the numbers it encodes, it should also contain the words $(r-1)^{+} \star(r-1)^{\omega}$ if $0^{\omega} \in L_{q}$, and $0^{+} 1 \star 0^{\omega}$ if $(r-1)^{\omega} \in L_{q}$.
} 
We thus proceed as follows. We consider a set $S \subseteq[0,1]$ that is both (resp. weakly) $r$ and $s$-recognizable, and assume by contradiction that $S$ has infinitely many boundary points. In Section 5.1, we derive some useful properties under this assumption. In Section 5.2, we then show that our assumption leads to a contradiction in the case of weak recognizability, proving that the sets that are both weakly $r$ - and weakly $s$-recognizable are necessarily definable in $\langle\mathbb{R}, \mathbb{Z},+,<\rangle$, hence Theorem 3.3. Finally, in Section 5.3, we show with the help of a counterexample that this result does not generalize to sets that are both $r$ - and $s$-recognizable.

5.1. Product stability. By hypothesis, the set $S \subseteq[0,1]$ is (resp. weakly) $r$-recognizable. Let $\mathcal{A}_{r}$ be a (resp. weak) RNA recognizing $S$ in base $r$. We assume w.l.o.g. that the transition relation of $\mathcal{A}_{r}$ is complete.

Since $S$ is $r$-recognizable, the set $B_{S}$ of boundary points of $S$ is $r$-recognizable by Lemma 4.1, Let $\mathcal{A}_{r}^{B}$ be a RNA recognizing $B_{S}$.

By assumption, $S$ has infinitely many boundary points, hence there exist infinitely many distinct paths of $\mathcal{A}_{r}^{B}$ that end up cycling in the same set of accepting states. One can thus extract from $\mathcal{A}_{r}^{B}$ an infinite language $L=0 \star u v^{*} t w^{\omega}$, where $t, u, v, w \in \Sigma_{r}^{*},|v|>0$, $|w|>0$, and $L$ encodes an infinite subset of the boundary points of $S$. We then define $y=\left[0 \star u v^{\omega}\right]_{r}$ and, for each $k \in \mathbb{N}_{>0}, y_{k}=\left[0 \star u v^{k} t w^{\omega}\right]_{r}$. The sequence $y_{1}, y_{2}, y_{3}, \ldots \in \mathbb{Q}^{\omega}$ forms an infinite sequence of distinct boundary points of $S$, converging to $y \in \mathbb{Q}$. If we have $y_{k}>y$ for infinitely many $k$, then we define $S^{1}=(S-y) \cap[0,1]$. Otherwise, we define $S^{1}=(-S+y) \cap[0,1]$. From Theorem [2.6, the set $S^{1}$ is both (resp. weakly) $r$ - and $s$-recognizable. Moreover, this set admits an infinite sequence of distinct boundary points that converges to 0 .

Let $\mathcal{A}_{r}^{1}$ and $\mathcal{A}_{s}^{1}$ be (resp. weak) RNA recognizing $S^{1}$ in the respective bases $r$ and $s$. The path $\pi_{0}$ of $\mathcal{A}_{r}^{1}$ that reads $0 \star 0^{\omega}$ is composed of a prefix labeled by $0 \star$, followed by an acyclic path of length $p \geq 0$, and finally by a cycle of length $q>0$. It follows that a word of the form $0 \star 0^{p} t$, with $t \in \Sigma_{r}^{\omega}$, is accepted by $\mathcal{A}_{r}^{1}$ iff the word $0 \star 0^{p+q} t$ is accepted as well. Remark that the set $S^{1}$ admits infinitely many boundary points with a base- $r$ encoding beginning with $0 \star 0^{p}$. Similar properties hold for $\mathcal{A}_{s}^{1}$. In this automaton, the path $\pi_{0}^{\prime}$ recognizing $0 \star 0^{\omega}$ reads the symbols 0 and $\star$, and then follows an acyclic sequence of length $p^{\prime}$ before reaching a cycle of length $q^{\prime}$.

We now define $S^{2}=r^{p} S^{1} \cap[0,1]$. Like $S^{1}$, the set $S^{2}$ admits an infinite sequence of boundary points that converges to 0 . Moreover, by Theorem 2.6, $S^{2}$ is both (resp. weakly) $r$ - and $s$-recognizable. Let $\mathcal{A}_{r}^{2}$ be a (resp. weak) RNA recognizing $S^{2}$ in base $r$. For every $t \in \Sigma_{r}^{\omega}$, the word $0 \star t$ is accepted by $\mathcal{A}_{r}^{2}$ iff the word $0 \star 0^{q} t$ is accepted as well. In other words, the fact that a number $x \in[0,1]$ belongs or not to $S^{2}$ is not influenced by the insertion of $q$ zero digits in its encodings, immediately after the symbol $\star$. This amounts to dividing the value of $x$ by $r^{q}$, which leads to the following definition.

Definition 5.1. Let $D \subseteq \mathbb{R}$ be a domain, and let $f \in \mathbb{R}_{>0}$. A set $S \subseteq D$ is $f$-product-stable in the domain $D$ iff for all $x \in D$ such that $f x \in D$, we have $x \in S \Leftrightarrow f x \in S$.

From the previous discussion, we have that $S^{2}$ is $r^{q}$-product-stable in $[0,1]$. We then define $S^{3}=s^{p^{\prime}} S^{2} \cap[0,1]$. The set $S^{3}$ is $r^{q}$-product-stable in [0,1] as well. By Theorem 2.6, $S^{3}$ is also both (resp. weakly) $r$ - and $s$-recognizable. Besides, since $S^{3}=r^{p} s^{p^{\prime}} S^{1} \cap[0,1]$, the set $S^{3}$ can alternatively be obtained by first defining $S^{4}=s^{p^{\prime}} S^{1} \cap[0,1]$, which is both (resp. weakly) $r$ - and $s$-recognizable by Theorem 2.6. Then, one has $S^{3}=r^{p} S^{4} \cap[0,1]$. By 
a similar reasoning in base $s$, we get that $S^{3}$ is $s^{q^{\prime}}$-product-stable in $[0,1]$. Like $S^{2}$, the set $S^{3}$ admits an infinite sequence of distinct boundary points that converges to 0 .

Finally, we replace the bases $r$ and $s$ by $r^{\prime}=r^{q}$ and $s^{\prime}=s^{q^{\prime}}$, thanks to Theorem 2.7. The results of this section are then summarized by the following lemma.

Lemma 5.2. Let $r, s \in \mathbb{N}_{>1}$ be two multiplicatively independent bases, and let $S \subseteq[0,1]$ be a set that is both (resp. weakly) $r$ - and s-recognizable, and that admits infinitely many boundary points. There exist powers $r^{\prime}=r^{i}$ and $s^{\prime}=s^{j}$ of $r$ and $s$, with $i, j \in \mathbb{N}_{>0}$, and a set $S^{\prime} \subseteq[0,1]$ that is both (resp. weakly) $r^{\prime}$ - and $s^{\prime}$-recognizable, both $r^{\prime}$ - and $s^{\prime}$-product-stable in $[0,1]$, and that admits infinitely many boundary points.

5.2. Recognizability by weak RNA. We are now ready to prove that our initial assumption that the set $S \subseteq[0,1]$ has infinitely many boundary points leads to a contradiction, under the hypothesis that $S$ is both weakly $r$-and weakly $s$-recognizable.

By Lemma 5.2, we can assume w.l.o.g. that $S$ is $r$ - and $s$-product-stable in $[0,1]$. Hence, there exist $\alpha, \beta \in(0,1]$ such that $\alpha \in S$ and $\beta \notin S$. For every $i, j \in \mathbb{Z}$ such that $r^{i} s^{j} \alpha \in(0,1]$, we thus have $r^{i} s^{j} \alpha \in S$. Similarly, for every $i, j \in \mathbb{Z}$ such that $r^{i} s^{j} \beta \in(0,1]$, we have $r^{i} s^{j} \beta \notin S$.

Let $\gamma$ be an arbitrary point in the open interval $(0,1)$. Since $r$ and $s$ are multiplicatively independent, it follows from Kronecker's approximation theorem [HW85] that any open interval of $\mathbb{R}_{>0}$ contains some number of the form $r^{i} / s^{j}$ with $i, j \in \mathbb{N}_{>0}$ [Per90]. Hence, for every sufficiently small $\varepsilon>0$ and $\delta \in\{\alpha, \beta\}$, there exist $i, j \in \mathbb{N}_{>0}$ such that

$$
0<\gamma-\varepsilon<\left(r^{i} / s^{j}\right) \delta<\gamma+\varepsilon<1
$$

showing that every neighborhood $N_{\varepsilon}(\gamma)$ of $\gamma$ contains one point from $S$ as well as one from $\bar{S}$. The latter property leads to a contradiction, since it implies that $S$ satisfies the dense oscillating sequence property, and therefore, by Theorem 2.3 , cannot be recognized by a weak RNA.

Taking into account the problem reductions introduced in Sections 4.1 and 4.2 , we thus have proven Theorem 3.3 .

Thanks to the above mentioned reductions, Theorem 3.3 has the following corollary. A set $S \subseteq \mathbb{R}$ is weakly $r$ - and weakly $s$-recognizable in two multiplicatively independent bases iff it can be expressed as a finite union $\bigcup_{i}\left(S_{i}^{I}+S_{i}^{F}\right)$, where each $S_{i}^{I} \subseteq \mathbb{Z}$ is of the form $S_{i}^{I}=\left\{a_{i}+k b_{i} \mid k \in \mathbb{N}\right\}$ with $a_{i}, b_{i} \in \mathbb{Z}$, and each $S_{i}^{F} \subseteq[0,1]$ is a finite union of intervals with rational extremities. It has already been observed in Wei99 that such a structural description of subsets of $\mathbb{R}$ is equivalent to definability in $\langle\mathbb{R}, \mathbb{Z},+,<\rangle$.

5.3. Recognizability by RNA. We now show that Theorem 3.3 does not directly generalize to non-weak recognizability. Indeed, a set can then be recognizable in two multiplicatively independent bases without being definable in $\langle\mathbb{R}, \mathbb{Z},+,<\rangle$. This property is established by the following theorem.

Theorem 5.3. For every pair of bases $r, s \in \mathbb{N}_{>1}$ that share the same set of prime factors, there exists a set $S$ that is both $r$-and s-recognizable, and that is not definable in the firstorder theory $\langle\mathbb{R}, \mathbb{Z},+,<\rangle$. 
Proof. A counterexample is provided by the set

$$
S=\left\{\frac{n}{f_{1}^{i_{1}} f_{2}^{i_{2}} \cdots f_{k}^{i_{k}}} \mid n \in \mathbb{Z}, i_{1}, i_{2}, \ldots, i_{k} \in \mathbb{N}\right\},
$$

where $f_{1}, f_{2}, \ldots f_{k}$ are the prime factors of $r$ and $s$.

In either base $t \in\{r, s\}$, this set is encoded by the language $L_{t}=\{0, t-1\} \Sigma_{t}^{*} \star \Sigma_{t}^{*}\left(0^{\omega} \cup\right.$ $\left.(t-1)^{\omega}\right)$, i.e., the set $S$ contains the numbers that admit dual encodings. Indeed, each word of $L_{t}$ represents a number $x=n / t^{k}(n \in \mathbb{Z}, k \in \mathbb{N})$ that belongs to $S$. Reciprocally, let $x$ be an element of $S$. One can assume w.l.o.g. that the denominator of $x$ is a power of $t$. Hence, $x$ admits an encoding that ends with $0^{\omega}$.

The language $L_{t}$ is clearly $\omega$-regular, hence $S$ is both $r$ - and $s$-recognizable. Suppose that $S$ is definable in $\langle\mathbb{R}, \mathbb{Z},+,<\rangle$. Then, it is weakly $t$-recognizable in any base $t$ thanks to Theorem 2.2. By Theorem 2.3 and since $S$ satisfies the dense oscillating sequence property, this leads to a contradiction.

Note that the set $S$ (resp. $\mathbb{R} \backslash S$ ) defined in the previous proof is recognizable by deterministic co-Büchi automata (resp. deterministic Büchi automata) in both bases $r$ and $s$. It follows that Theorem 3.3 does not generalize to sets recognizable by those classes of automata either.

The case of bases that do not share the same set of prime factors is investigated in the next section.

\section{BASES With DiffERENT SETS OF PRIME FACTORS}

We now consider two bases $r, s \in \mathbb{N}_{>1}$ that do not share the same set of prime factors. Since this property implies that $r$ and $s$ are multiplicatively independent, we know by Theorem 3.3 that any subset of $\mathbb{R}$ that is simultaneously weakly $r$ - and weakly $s$-recognizable must be definable in $\langle\mathbb{R}, \mathbb{Z},+,<\rangle$.

The goal of this section is now to prove Theorem 3.4, i.e., that a subset of $\mathbb{R}$ that is both $r$ - and $s$-recognizable is necessarily definable in $\langle\mathbb{R}, \mathbb{Z},+,<\rangle$. Recall that, as shown in Section 5.3 , this result does not extend to pairs of bases that are multiplicatively independent but share the same prime factors.

We proceed like in Section 5 and start from an arbitrary set $S$ that is both $r$ - and $s$-recognizable. Thanks to the reduction discussed in Section 4.1, it suffices to consider $S \subseteq$ $[0,1]$. Moreover, according to Lemma 4.2, one can prove that $S$ is definable in $\langle\mathbb{R}, \mathbb{Z},+,<\rangle$ by showing that it admits only finitely many boundary points. We thus assume that $S$ admits infinitely many boundary points. From this assumption, we will derive in Sections 6.1 and 6.2 additional properties that will eventually lead to a contradiction.

It is possible to reuse part of the reasoning made in Section 5 , By Lemma 5.2, there exist bases $r^{\prime}$ and $s^{\prime}$ with different sets of prime factors, and a set $S^{\prime} \subseteq[0,1]$ that is both $r^{\prime}$ - and $s^{\prime}$-recognizable, both $r^{\prime}$ - and $s^{\prime}$-product-stable in $[0,1]$, and that has infinitely many boundary points. Replacing the set $S$ by $S^{\prime}$, and the bases $r, s$ by $r^{\prime}, s^{\prime}$, we can thus assume w.l.o.g. that the set $S$ that we consider is both $r$ - and $s$-product-stable in [0,1]. Finally, we also impose w.l.o.g. that there exists a prime factor of $s$ that does not divide $r$. 
6.1. Sum stability. Our first strategy consists in exploiting Cobham's theorem so as to derive additional properties of $S$. The initial step is to build from $S$ a set $S^{\prime} \subseteq \mathbb{R}_{\geq 0}$ that coincides with $S$ over [0,1], shares the same recognizability and product-stability properties, and contains numbers with non-trivial integer parts.

Lemma 6.1. Let $r, s \in \mathbb{N}_{>1}$ be two bases with different sets of prime factors, and let $S \subseteq[0,1]$ be a set that is $r$ - and $s$-recognizable, $r$ - and s-product-stable in $[0,1]$, and that has infinitely many boundary points. There exists a set $S^{\prime} \subseteq \mathbb{R}_{\geq 0}$ that is $r$-and s-recognizable, $r$ - and s-product-stable in $\mathbb{R}_{\geq 0}$, and that has infinitely many boundary points.

Proof. Let $S^{\prime}=\left\{r^{k} x \mid x \in S \wedge k \in \mathbb{N}\right\}$. This set is clearly $r$-product-stable in $\mathbb{R}_{\geq 0}$. Since $S$ is $r$-product-stable in $[0,1]$, we have $S^{\prime} \cap[0,1]=S$ showing that $S^{\prime}$ has infinitely many boundary points. A RNA $\mathcal{A}_{r}^{\prime}$ recognizing $S^{\prime}$ in base $r$ is built from an automaton $\mathcal{A}_{r}$ recognizing $S$ by delaying arbitrarily the reading of the symbol $\star$. In other words, a word $u v \star w$ is accepted by $\mathcal{A}_{r}^{\prime}$, with $u \in\{0, r-1\} \Sigma_{r}^{*}, v \in \Sigma_{r}^{*}$, and $w \in \Sigma_{r}^{\omega}$, whenever the word $u \star v w$ is accepted by $\mathcal{A}_{r}$.

In order to prove that $S^{\prime}$ is $s$-recognizable, notice that, since $S$ is both $r$ - and $s$-productstable in [0,1], we have $S^{\prime}=\left\{r^{i} s^{j} x \mid x \in S \wedge i, j \in \mathbb{Z}\right\}$. The set $S^{\prime}$ can therefore be expressed as $S^{\prime}=\left\{s^{k} x \mid x \in S \wedge k \in \mathbb{N}\right\}$. By the same reasoning as in base $r$, this set is $s$-recognizable, as well as $s$-product-stable in $\mathbb{R}_{\geq 0}$.

Consider now a set $S^{\prime}$ obtained from $S$ by Lemma 6.1. As discussed in Section 4.1. this set can be expressed as a finite union $S^{\prime}=\bigcup_{i}\left(S_{i}^{I}+S_{i}^{F}\right)$, where for each $i$, we have $S_{i}^{I} \subseteq \mathbb{N}$ and $S_{i}^{F} \subseteq[0,1]$. Moreover, for each $i$, the set $S_{i}^{I}$ is both $r$ - and $s$-recognizable, and it follows from Theorem 3.1 that this set is definable in $\langle\mathbb{N},+,<\rangle$. Since such a set is ultimately periodic [Cob69, BHMV94, there exists $n_{i} \in \mathbb{N}_{>0}$ for which $\forall x \in \mathbb{N}, x \geq n_{i}: x \in S_{i}^{I} \Leftrightarrow$ $x+n_{i} \in S_{i}^{I}$. By defining $n=\operatorname{lcm}_{i}\left(n_{i}\right)$, we obtain $\forall x \in \mathbb{R}_{\geq 0}, x \geq n: x \in S^{\prime} \Leftrightarrow x+n \in S^{\prime}$. This prompts the following definition.

Definition 6.2. Let $D \subseteq \mathbb{R}$ be a domain, and let $t \in \mathbb{R}$. A set $S \subseteq D$ is $t$-sum-stable in $D$ iff for all $x \in D$ such that $x+t \in D$, we have $x \in S \Leftrightarrow x+t \in S$.

Let us show that the set $S^{\prime \prime}=(1 / n) S^{\prime} \backslash\{0\}$ is 1 -sum-stable in $\mathbb{R}_{>0}$. For every $x \geq 1$, we have $x \in S^{\prime \prime} \Leftrightarrow x+1 \in S^{\prime \prime}$. For $x<1$, we choose $k \in \mathbb{N}$ such that $r^{k} x \geq 1$. Exploiting the properties of $S^{\prime}$ (transposed to $S^{\prime \prime}$ ), we get $x \in S^{\prime \prime} \Leftrightarrow r^{k} x \in S^{\prime \prime} \Leftrightarrow r^{k} x+r^{k} \in S^{\prime \prime} \Leftrightarrow x+1 \in$ $S^{\prime \prime}$. Lemma 6.1 can thus be refined as follows.

Lemma 6.3. Let $r, s \in \mathbb{N}_{>1}$ be two bases with different sets of prime factors, and let $S \subseteq[0,1]$ be a set that is $r$ - and $s$-recognizable, $r$ - and s-product-stable in $[0,1]$, and that has infinitely many boundary points. There exists a set $S^{\prime} \subseteq \mathbb{R}_{>0}$ that is $r$-and s-recognizable, has infinitely many boundary points, and is r-product-, s-product- and 1-sum-stable in $\mathbb{R}_{>0}$.

Note that Lemmas 6.1 and 6.3 still hold if the bases $r$ and $s$ are multiplicatively independent.

6.2. Exploiting sum-stability properties. Consider a set $S^{\prime} \subseteq \mathbb{R}_{>0}$ that satisfies the properties expressed by Lemma 6.3. It remains to show that these properties lead to a contradiction. The hypothesis on the prime factors of $r$ and $s$ is explicitly used in this section.

We proceed by characterizing the numbers $t \in \mathbb{R}$ for which $S^{\prime}$ is $t$-sum-stable in $\mathbb{R}_{>0}$. These form the set $T_{S^{\prime}}=\left\{t \in \mathbb{R} \mid \forall x \in \mathbb{R}_{>0}: x+t \in \mathbb{R}_{>0} \Rightarrow\left(x \in S^{\prime} \Leftrightarrow x+t \in S^{\prime}\right)\right\}$. Since 
$S^{\prime}$ is $r$-recognizable, it is definable in $\left\langle\mathbb{R}, \mathbb{Z},+,<, X_{r}\right\rangle$ by Theorem 2.1 , and so is $T_{S^{\prime}}$, that is therefore $r$-recognizable as well.

The set $T_{S^{\prime}}$ enjoys interesting closure properties:

Property 6.4. For every $t, u \in T_{S^{\prime}}$ and $a, b \in \mathbb{Z}$, we have $a t+b u \in T_{S^{\prime}}$.

The set $T_{S^{\prime}}$ is also $r$ - and $s$-product stable in $\mathbb{R}$. Since $1 \in T_{S^{\prime}}$, this yields the following property.

Property 6.5. For every $k \in \mathbb{Z}$, we have $r^{k} \in T_{S^{\prime}}$ and $s^{k} \in T_{S^{\prime}}$.

Intuitively, being able to add or subtract $r^{k}$ from a number, for any $k$, makes it possible to change in an arbitrary way finitely many digits in its base- $r$ encodings, without influencing the fact that this number belongs or not to $S^{\prime}$. Our next step will be to show that this property can be extended to all digits of base- $r$ encodings, implying either $S^{\prime}=\emptyset$ or $S^{\prime}=\mathbb{R}_{>0}$. This would then contradict our assumption that $S^{\prime}$ has infinitely many boundary points.

Lemma 6.6. Let $r, s \in \mathbb{N}_{>1}$ be two bases such that $s$ has a prime factor that does not divide $r$. The lengths of the smallest periods of the base-r encodings of $1 / s^{k}$ are unbounded w.r.t. $k$.

Proof. The base- $r$ encodings of $1 / s^{k}$ are of the form $0^{+} \star v_{k} u_{k}^{\omega}$, with $v_{k} \in \Sigma_{r}^{*}$ and $u_{k} \in \Sigma_{r}^{+}$. We have

Hence,

$$
\begin{gathered}
\frac{r^{\left|v_{k}\right|}}{s^{k}}=\left[0 v_{k} \star u_{k}^{\omega}\right]_{r}, \\
\frac{r^{\left|v_{k}\right|+\left|u_{k}\right|}}{s^{k}}=\left[0 v_{k} u_{k} \star u_{k}^{\omega}\right]_{r} .
\end{gathered}
$$

$$
\frac{1}{s^{k}}=\frac{a_{k}}{r^{\left|v_{k}\right|}\left(r^{\left|u_{k}\right|}-1\right)}
$$

with $a_{k}=\left[v_{k} u_{k}\right]_{r}-\left[v_{k}\right]_{r} \in \mathbb{N}_{>0}$.

It follows that the lengths $\left|u_{k}\right|$ and $\left|v_{k}\right|$ are the smallest naturals such that $s^{k}$ divides $r^{\left|v_{k}\right|}\left(r^{\left|u_{k}\right|}-1\right)$. By hypothesis, there exists a prime factor $f$ of $s$ that does not divide $r$. This implies that the lengths of the periods $u_{k}$ must be unbounded w.r.t. $k$.

Property 6.7. There exist $l, m \in \mathbb{N}_{>0}$ such that, for every $k \in \mathbb{N}_{>0}$, we have

$$
\frac{m}{r^{l k}-1} \in T_{S^{\prime}}
$$

Proof. By Property 6.5, we have $1 / s^{k} \in T_{S^{\prime}}$ for all $k \in \mathbb{N}$. From Lemma 6.6, the lengths of the smallest periods $u_{k}$ of the base- $r$ encodings of $1 / s^{k}$ are unbounded w.r.k. $k$.

Consider a RNA $\mathcal{A}_{r}^{T}$ recognizing $T_{S^{\prime}}$ in base $r$. We study the rational numbers accepted by $\mathcal{A}_{r}^{T}$, which have base- $r$ encodings of the form $v \star w u^{\omega}$. We assume w.l.o.g. that the considered periods $u$ are the shortest possible ones. It follows from the unboundedness of $u_{k}$ that $T_{S^{\prime}}$ contains rational numbers with infinitely many distinct periods. RNA are deterministic Muller automata; hence, their accepting conditions are finite unions of subsets of their set of states. An infinite number of encodings of rationals with distinct periods thus end in exactly the same subset of accepting states. In particular, there exist $u, u^{\prime}, v, v^{\prime}, w, w^{\prime}$ such that $u^{\omega}$ is not a suffix of $\left(u^{\prime}\right)^{\omega}$, the words $v \star w u^{\omega}$ and $v^{\prime} \star w^{\prime}\left(u^{\prime}\right)^{\omega}$ are both accepted by 
$\mathcal{A}_{r}^{T}$, and the paths $\pi$ and $\pi^{\prime}$ of $\mathcal{A}_{r}^{T}$ reading them end up cycling in exactly the same subset of accepting states.

Let $q$ be one of these states, and $u_{1}, u_{2} \in \Sigma_{r}^{+}$be periods of the (respective) words read by $\pi$ and $\pi^{\prime}$ after reaching $q$ in their final cycle. These periods can be repeated arbitrarily, hence we can assume w.l.o.g. that $\left|u_{1}\right|=\left|u_{2}\right|$. Moreover we can assume w.l.o.g. that $\left[u_{2}\right]_{r}>\left[u_{1}\right]_{r}$, otherwise $u^{\omega}$ would be a suffix of $\left(u^{\prime}\right)^{\omega}$. Besides, there exist $v, w \in \Sigma_{r}^{*}$ such that $v \star w$ reaches $q$. From the structure of $\mathcal{A}_{r}^{T}$, it follows that for every $k \geq 0$, the word $v \star w\left(u_{1}^{k} u_{2}\right)^{\omega}$ is accepted by $\mathcal{A}_{r}^{T}$.

For each $k \geq 0$, we thus have $\left[v \star w\left(u_{1}^{k} u_{2}\right)^{\omega}\right]_{r} \in T_{S^{\prime}}$. Developing, we get

$$
\frac{d_{k}+\left[v w \star 0^{\omega}\right]_{r}}{r^{|w|}} \in T_{S^{\prime}},
$$

with $d_{k}=\left[\star\left(u_{1}^{k} u_{2}\right)^{\omega}\right]_{r}$. Thanks to Properties 6.4 and 6.5, and the $r$-product-stability property of $T_{S^{\prime}}$, this implies $d_{k} \in T_{S^{\prime}}$. We now express $d_{k}$ in terms of $\left[u_{1}\right]_{r},\left[u_{2}\right]_{r}$, and $k$ :

$$
d_{k}=\frac{\left[u_{1}^{k} u_{2}\right]_{r}}{r^{l(k+1)}-1}=\frac{\left[u_{2}\right]_{r}-\left[u_{1}\right]_{r}}{r^{l(k+1)}-1}+\frac{\left[u_{1}\right]_{r}}{r^{l}-1}, \text { where } l=\left|u_{1}\right|=\left|u_{2}\right| .
$$

The next step will consist in getting rid of the second term of this expression. By Properties 6.4 and 6.5. we have for all $k \in \mathbb{N}$,

$$
\left(r^{l}-1\right) d_{k}-\left[u_{1}\right]_{r}=\frac{m}{r^{l(k+1)}-1} \in T_{S^{\prime}},
$$

where $m=\left(r^{l}-1\right)\left(\left[u_{2}\right]_{r}-\left[u_{1}\right]_{r}\right)$ is such that $m \in \mathbb{N}_{>0}$. For all $k>0$, we thus have

$$
\frac{m}{r^{l k}-1} \in T_{S^{\prime}}
$$

We are now ready to conclude. Given $l$ and $m$ by Property 6.7 we define $S^{\prime \prime}=(1 / m) S^{\prime}$. Like $S^{\prime}$, this set has infinitely many boundary points. The set $T_{S^{\prime \prime}}$ of the values $t$ for which $S^{\prime \prime}$ is $t$-sum-stable in $\mathbb{R}_{>0}$ is given by $T_{S^{\prime \prime}}=(1 / m) T_{S^{\prime}}$. This set is thus $r$-recognizable. From Properties 6.4 and 6.5, we have for every $k \in \mathbb{N}, 1 / r^{k} \in T_{S^{\prime \prime}}$. Finally, from Property 6.7, we have for every $k>0$,

$$
\frac{1}{r^{l k}-1} \in T_{S^{\prime \prime}}
$$

Property 6.8. The set $T_{S^{\prime \prime}}$ is equal to $\mathbb{R}$.

Proof. Since $T_{S^{\prime \prime}}$ and $\mathbb{R}$ are both $r$-recognizable, and two $\omega$-regular languages are equal iff they share the same subset of ultimately periodic words [PP04], it is actually sufficient to show that $T_{S^{\prime \prime}} \cap \mathbb{Q}=\mathbb{Q}$. Every rational $t$ admits a base- $r$ encoding of the form $v \star w u^{\omega}$, where $|u|=l k$ for some $k \in \mathbb{N}_{>0}$. We have

$$
t=\frac{\left[v w \star 0^{\omega}\right]_{r}}{r^{|w|}}+\frac{[u]_{r}}{r^{|w|}\left(r^{l k}-1\right)} .
$$

Since $1 / r^{|w|} \in T_{S^{\prime \prime}}$ and $1 /\left(r^{l k}-1\right) \in T_{S^{\prime \prime}}$, the closure and product-stability properties of $T_{S^{\prime \prime}}$ imply $t \in T_{S^{\prime \prime}}$. 
As a consequence, we either have $S^{\prime \prime}=\emptyset$ or $S^{\prime \prime}=\mathbb{R}_{>0}$, which contradicts our initial assumption that this set has infinitely many boundary points. As a consequence, our original set $S$ is definable in $\langle\mathbb{R}, \mathbb{Z},+,<\rangle$, and we have proven Theorem 3.4 .

\section{Conclusions}

In this article, we have established that the sets of real numbers that can be recognized by finite automata in two sufficiently different bases are exactly those that are definable in the first-order additive theory of real and integer variables $\langle\mathbb{R}, \mathbb{Z},+,<\rangle$. In the case of weak deterministic automata, used in actual implementations of symbolic representation systems LASH, FAST, LIRA, the condition on the bases turns out to be multiplicative independence. It is worth mentioning that recognizability in multiplicatively dependent bases is equivalent to recognizability in one of them, and that definability in $\langle\mathbb{R}, \mathbb{Z},+,<\rangle$ implies recognizability in every base. We have thus obtained a complete characterization of the sets of numbers recognizable in multiple bases, similar to the one known for the integer domain Cob69.

For Muller, deterministic Büchi, and co-Büchi automata, we have demonstrated that multiplicative independence of the bases is not a strong enough condition, and that the bases must have different sets of prime factors in order to force definability of the represented sets in $\langle\mathbb{R}, \mathbb{Z},+,<\rangle$. Recall that the sets definable in that theory can all be recognized by weak deterministic automata. We have thus established that the sets of real numbers that can be recognized by infinite-word automata in all encoding bases are exactly those that are recognizable by weak deterministic automata.

It is worth mentioning that, prior to this result, weak deterministic automata were already been used as actual data structures for representing sets of real numbers in statespace exploration tools [BJW05, LASH]. The motivation behind their use was at this time essentially practical: The algorithmic manipulation of these automata was considerably simpler than that of unrestricted infinite-word ones. Moreover, their expressive power was known to be sufficient for handling the sets definable in $\langle\mathbb{R}, \mathbb{Z},+,<\rangle$, which matched the application requirements. The results developed in this article now bring an additional theoretical justification to the choice of weak deterministic automata for representing sets of real and integer numbers: If recognizability by automata has to be achieved regardless of the representation base, then the representable sets are exactly those that can be recognized by weak deterministic automata.

\section{REFERENCES}

[BB09] B. Boigelot and J. Brusten. A generalization of Cobham's theorem to automata over real numbers. Theoretical Computer Science, 410(18):1694 - 1703, 2009.

[BBR97] B. Boigelot, L. Bronne, and S. Rassart. An improved reachability analysis method for strongly linear hybrid systems. In Proc. 9th CAV, volume 1254 of Lecture Notes in Computer Science, pages 167-177, Haifa, June 1997. Springer.

[BHMV94] V. Bruyère, G. Hansel, C. Michaux, and R. Villemaire. Logic and p-recognizable sets of integers. Bulletin of the Belgian Mathematical Society, 1(2):191-238, March 1994.

[BJW05] B. Boigelot, S. Jodogne, and P. Wolper. An effective decision procedure for linear arithmetic over the integers and reals. ACM Transactions on Computational Logic, 6(3):614-633, 2005.

[Boi98] B. Boigelot. Symbolic methods for exploring infinite state Sspaces. PhD thesis, Université de Liège, 1998. 
[BRW98] B. Boigelot, S. Rassart, and P. Wolper. On the expressiveness of real and integer arithmetic automata. In Proc. 25th ICALP, volume 1443 of Lecture Notes in Computer Science, pages 152-163, Aalborg, July 1998. Springer.

[Büc62] J. R. Büchi. On a decision method in restricted second order arithmetic. In Proc. International Congress on Logic, Methodoloy and Philosophy of Science, pages 1-12, Stanford, 1962. Stanford University Press.

[Cob69] A. Cobham. On the base-dependence of sets of numbers recognizable by finite automata. Mathematical Systems Theory, 3:186-192, 1969.

[EK06] J. Eisinger and F. Klaedtke. Don't care words with an application to the automata-based approach for real addition. In Proc. 18th CAV, volume 4144 of Lecture Notes in Computer Science, pages 67-80, Seattle, August 2006. Springer.

[FAST] Fast Acceleration of Symbolic Transition systems (FAST). Available at : http://www.lsv.ens-cachan.fr/fast/.

[HW85] G. H. Hardy and E. M. Wright. An introduction to the theory of numbers. Oxford University Press, 5th edition, 1985.

[LASH] The Liège Automata-based Symbolic Handler (LASH). Available at : http://www.montefiore.ulg.ac.be/ boigelot/research/lash/.

[LIRA] Linear Integer/Real Arithmetic solver (LIRA). Available at : http://lira.gforge.avacs.org/.

[McN66] R. McNaughton. Testing and generating infinite sequences by a finite automaton. Information and Control, 9(5):521-530, 1966.

[MS97] O. Maler and L. Staiger. On syntactic congruences for $\omega$-languages. Theoretical Computer Science, 183(1):93-112, 1997.

[Per90] D. Perrin. Finite automata. In J. van Leeuwen, editor, Handbook of Theoretical Computer Science, Volume B: Formal Models and Semantics, pages 1-57. Elsevier and MIT Press, 1990.

[PP04] D. Perrin and J.E. Pin. Infinite words, volume 141 of Pure and Applied Mathematics. Elsevier, 2004.

[Saf88] S. Safra. On the complexity of $\omega$-automata. In Proc. 29th Symposium on Foundations of Computer Science, pages 319-327. IEEE Computer Society, October 1988.

[Var07] M. Vardi. The Büchi complementation saga. In Proc. 24th. STACS, volume 4393 of Lecture Notes in Computer Science, pages 12-22, Aachen, February 2007. Springer.

[WB95] P. Wolper and B. Boigelot. An automata-theoretic approach to Presburger arithmetic constraints. In Proc. 2nd SAS, volume 983 of Lecture Notes in Computer Science, pages 21-32, Glasgow, September 1995. Springer.

[Wei99] V. Weispfenning. Mixed real-integer linear quantifier elimination. In Proc. ACM SIGSAM IS$S A C$, pages 129-136, Vancouver, July 1999. ACM Press.

[Wil93] T. Wilke. Locally threshold testable languages of infinite words. In Proc. 10th STACS, volume 665 of Lecture Notes in Computer Science, pages 607-616, Würzburg, 1993. Springer.

This work is licensed under the Creative Commons Attribution-NoDerivs License. To view a copy of this license, visit http://creativecommons.org/licenses/by-nd/2.0/ or send a letter to Creative Commons, 171 Second St, Suite 300, San Francisco, CA 94105, USA, or Eisenacher Strasse 2, 10777 Berlin, Germany 